\title{
Sevoflurane causes cognitive impairment by inducing iron deficiency and inhibiting proliferation of neural precursor cells in infant mice
}

\section{Jinhong Xie}

Hebei Normal University

\section{Yong Zuo}

Hebei Normal University

\section{Xue Zhang}

Hebei Normal University

Anand Thirupathi

Ningbo University

Di Zhang

Hebei Normal University

Jianhua Zhang

Hebei Normal University

Zhenhua Shi ( $\nabla$ shizhhtom@126.com )

Hebei Normal University https://orcid.org/0000-0001-7308-3239

\section{Research Article}

Keywords: Sevoflurane, cognitive impairment, iron metabolism, neural progenitor cells, neural stem cells, neurons

Posted Date: February 7th, 2022

DOI: https://doi.org/10.21203/rs.3.rs-1297590/v1

License: (c) (1) This work is licensed under a Creative Commons Attribution 4.0 International License. Read Full License 


\section{Abstract}

Anesthesia can induce cognitive impairment in young rodents. However, the exact mechanism is not clear. Iron deficiency can cause neurodevelopment disorder. Therefore, we investigated the effect of iron deficiency induced by sevoflurane (Sev) on cognitive function in infant mice. C57BL/ 6 mice of postnatal day 14 and neural stem cells (NSCs) NE4C were treated with $2 \%$ Sev for 6 h. We used the Morris water maze (MWM) to test the cognitive function of infant mice. Then, we used immunohistochemical stain, immunofluorescence assay, western blot, and flow cytometer to evaluate the myelinogenesis, iron levels, cell proliferation in cortex and hippocampus or in NE4C cells in vivo or in vitro. We found that Sev significantly caused cognitive deficiency. Further, we found that Sev inhibited the oligodendrocytes proliferation and myelinogenesis by decreasing MBP and CC-1 expression and iron levels. Meanwhile, Sev also induced the iron deficiency in neurons, and NSCs by down-regulating FtH and FtL expression and up-regulating the TfR 1 expression in the cortex and hippocampus, which dramatically suppressed the proliferation of NSCs and neural progenitor cells(NPCs) by decreasing the expression of Pax6 and Ki67, and caused the decrease of counts of neurons. Interestingly, iron supplementation before anesthesia significantly improved iron deficiency and cognitive deficiency induced by Sev in infant mice. In conclusion, these data suggested that Sev-induced iron deficiency might be a new mechanism of cognitive impairment caused by inhaled anesthetics in infant mice. The iron supplementation before anesthesia is an effective strategy to prevent cognitive impairment caused by Sev in infants.

\section{Introduction}

Millions of children under 3 years of age requires general anesthesia each year for various reasons [1]. Anesthetics are drugs that are used during surgery. Despite their proven safety, the neurotoxicity induced by anesthetics, especially in infants or young children, has raised wide concerns [2, 3]. The neurotoxicity was mainly manifested as cognitive impairment, so infants are at risk of poor neurocognitive outcomes after general anesthesia for many reasons. Therefore, the US Food and Drug Administration (FDA) had released a new warning regarding the use of anesthetics in children under 3 years of age [4].

Sev is a commonly used anesthetic in pediatric surgery. Infant brain development plasticity is strong, sensitive to external influencing factors including anesthetics. It was reported that Sev could cause the long-term diminution of cognition and regional volumetric alterations in brain structure in early childhood which was associated with brain development $[1,5]$. Our previous and other studies showed that Sev could inhibite the myelin sheath formation when the fetus or young mice were exposed to Sev, inducing cognitive dysfunction [6, 7]. Neonatal to anesthesia lead to cognitive deficits in old age [8]. In addition, exposure of young animals, including both rodents and primates (including humans), to general anesthesia causes nerve cell apoptosis $[8,9]$ which further affected the brain development.

Iron, as a cofactor of many enzymes, was one of the essential trace elements for human beings which was involved in many metabolic processes in the central nervous system, including energy production through electronic respiratory chain, DNA synthesis, and oxygen transport[10, 11]. Hence, maintaining iron 
homeostasis played an important role in infant brain development. Ferritin is a type of iron storage protein that includes two subunits heavy chain ferritin $(\mathrm{FtH})$ and light chain ferritin (FtL). Transferrin receptor1 (TfR1) contributed to the iron ions in cells. The expressions of ferritin and TfR1 were regulated by cellular iron concentration via IRE-IRP system. When cellular iron levels increased, ferritin expression upregulated and TfR1 expression downregulated. On the contrary, ferritin expression downregulated and TfR1 expression upregulated. Iron was also an important component of the electronic respiratory chain. G. Xu et al. reported that Sev could cause the mitochondrial dysfunction inducing the cognitive deficiency in young mice [12]. Our previous experiments showed that Sev could cause the iron homeostasis disorder to induce neurotoxicity in elder mice, and higher iron levels further affected the function of mitochondrial and ATP production $[13,14]$.

The functional damage of Sev on neurons is closely related to the cognitive impairment induced by Sev. Some studies showed that Sev contributed to neurotoxicity by inducing apoptosis and inflammation [15, 16]. NSCs and NPCs have the potential to differentiate into neurons, astrocytes, and oligodendrocytes to produce a large number of mature brain cells. A recent study showed that Sev also affected the neurogenesis, differentiation to relieve the brain development through inhibiting cell cycle, DNA methylation of NSCs in fetal or young mice. $[17,18]$.

These findings suggested a functional connection between NSCs or neurons damage and Sev. However, it remains largely unknown how Sev could affect the NSCs growth and differentiation and further damage neuron function, inducing cognitive impairment in young mice. Considering the role of iron in growth and differentiation, we tested the hypothesis that Sev could cause a cognitive impairment via dysfunction of iron metabolism in NSCs and neurons. We found that Sev caused the iron deficiency in NSCs, oligodendrocytes and neurons which inhibited the neural development, inducing cognitive impairment. Iron supplementation before anesthesia is an effective strategy to prevent cognitive impairment caused by Sev.

\section{Methods}

\section{Experimental design, mice anesthesia, BrdU treatment and iron therapy}

All animal experiments were performed in accordance with ethical standards and the procedures were approved by the Hebei normal university ethics committee. Postnatal 14 days C57BL/ 6 male mice were fed in stainless steel rust-free cages at $22-24^{\circ} \mathrm{C}$ and a 12 -h light/dark cycle and were provided free access to the food and distilled water. The mice were assigned into four groups: control group, Sev treatment group, iron treatment group, Sev+iron treatment group. Anesthesia treatment was according to our previous report[6]. Briefly, the mice in the anesthesia groups were placed in an anesthetic induction chamber filled with Sev (2\%) for approximately $5 \mathrm{~min}$ until they became unconscious. The mice were then removed and attached to one of the nose cones of the anesthetizing apparatus in order to be exposed to the same amount of anesthetic. The mice were treated with gases of $2 \% \mathrm{Sev}$ and $100 \%$ oxygen within the anesthetic chamber for 6 hours and were continuously monitored (Ohmeda Excel 210 SE anesthetic 
machine, Datex Instrumentarium Corp., Helsinki, Finland). BrdU labeling, behavioral test, and iron therapy of mice are shown in Figure 1.

\section{Cell culture and anesthesia}

NE4C cells, which were neuron stem cells from mouse, were grown in MEM supplemented with $10 \%$ fetal bovine serum, $100 \mathrm{U} / \mathrm{ml}$ of penicillin, and $100 \mathrm{mg} / \mathrm{ml}$ of streptomycin. Cells were maintained at $37^{\circ} \mathrm{C}$ in a humidified $5 \% \mathrm{CO}_{2} / 95 \%$ air incubator. To investigate the effect of Sev on the NE4C cells, when the cells were in logarithmic phase, we treated the NE4C cells with $2 \%$ Sev for $6 \mathrm{~h}$ in an anesthetic chamber in a carbon dioxide incubator with $5 \% \mathrm{CO} 2$ at $37^{\circ} \mathrm{C}$. Then the cells were harvested to be assayed.

\section{Morris water maze (MWM) test}

MWM test was done according to our previous report [6]. Briefly, for mice in control group, each mouse was given swimming training one time a day for 6 days. For Sev group mice, the mice were first anesthetized and then they were given the MWM test. Then, these mice were given swimming training one time a day for 6 days. On the sixth day, the platform was removed to test the times of crossing platform. For each mouse, the escape latency was recorded as a maximum of $120 \mathrm{~s}$.

\section{Immunohistochemistry studies and immunofluorescence assay}

For immunohistochemistry assay, the brains were carefully dissected, removed, post-fixed, and then transferred to $30 \%$ sucrose for 2 days. The coronal brain sections were cut and frozen with a thickness of $15 \mu \mathrm{m}$, and then sections were washed with $0.01 \mathrm{M}$ PBS three times for 5 min each time. The sections were incubated with $3 \%$

$\mathrm{H} 2 \mathrm{O} 2$ for 20 min and then were washed with $0.01 \mathrm{M}$ PBS three times for 5 min each time. The sections were then incubated with goat serum at $37^{\circ} \mathrm{C}$ for $60 \mathrm{~min}$. The samples were incubated overnight at $4{ }^{\circ} \mathrm{C}$ with the rabbit polyclonal anti-MBP antibody (Cat. No.10458-1-AP, Proteintech, Wuhan, China) and antiCC1 antibody (1:250; Cat. No. ab16794, Abcam, Massachusetts, USA). Next, they were incubated with biotinylated secondary goat anti-rabbit IgG at $37^{\circ} \mathrm{C}$ for $1 \mathrm{~h}$. After being washed with PBS three times for 5 min each time, the sections were incubated in horseradish peroxidase (HRP)-labeled streptavidin reagent for $1 \mathrm{~h}$ at room temperature and then were stained using a DAB kit (SK-4100; VECTOR, Kronshagen, Germany) for $40 \mathrm{~s}$. Finally, the sections were dehydrated and mounted. Images were captured using a ZEISS LSM710 (LSM710; ZEISS, Germany).

For the immunofluorescence assay, mouse monoclonal anti-NeuN antibody (1:200; Cat. No. ab104224, Abcam, Massachusetts, USA), rabbit monoclonal anti-FtL (1:400; Cat. No. ab109373, Abcam, Massachusetts, USA), and rabbit monoclonal anti-FtH (1:400; Cat. No. ab183781, Abcam, Massachusetts, USA) were used as primary antibodies. FITC-conjugated and rhodamine-conjugated secondary antibodies were used. Images were captured using a ZEISS LSM710 (LSM710; ZEISS, Germany).

\section{Perl's iron staining}


The brain slices were washed with $0.01 \mathrm{~mol} / \mathrm{L} \mathrm{PBS}(\mathrm{pH}$ 7.4) for three times, for 5 min each, and then the slices were incubated with $3 \% \mathrm{H} 2 \mathrm{O} 2$ for $20 \mathrm{~min}$. The slices were washed with $0.01 \mathrm{~mol} / \mathrm{L} \mathrm{PBS} \mathrm{(pH7.4)} \mathrm{for}$ three times, 5 min each again. The slices were stained using Perl's dye liquor for $8 \mathrm{~h}$ and then were washed with $0.01 \mathrm{~mol} / \mathrm{L}$ PBS (pH7.4) for three times, 5 min each. Finally, the slices were strengthened using dyeing with $\mathrm{DAB}-\mathrm{H} 2 \mathrm{O} 2$ solution and then were washed by double-distilled water for $30 \mathrm{~min}$. After being washed with gradient of ethanol and xylene, the slices were packaged by neutral gum, and the images were taken with the microscope (ZEISS Axio imager).

\section{Western blot analysis}

The hippocampal and cortex tissues were homogenized in RIPA buffer followed by centrifugation at $12,000 \mathrm{~g}$ for $20 \mathrm{~min}$ at $4{ }^{\circ} \mathrm{C}$. The supernatant containing proteins was collected and its was measured content using protein quantification kit (KangWei, Beijing, China). The samples were resolved by $8-12 \%$ of SDS-PAGE, respectively, and then transferred to nitrocellulose membranes (Millipore, Bedford, MA, USA). The target proteins FtH, FtL, NeuN, Ki67, and CC1 (Abcam, USA), MBP (Proteintech, Wuhan, China), TfR1 (ThermoFisher Scientific, USA) \Pax6 (Biolegend, USA), IBA1, and GFAP (Millipore, USA) were detected by their primary antibodies. The relative expression quantity of proteins was normalized to that of $\beta$-actin (KangWei, Beijing, China).

\section{Statistical analysis}

All experiments were performed at least in triplicate. One-way ANOVA or t-test was used to estimate the overall significance, followed by the post hoc Tukey's test corrected for multiple comparisons. The data are presented as means \pm standard error of mean $(S E M)$. A probability level of $95 \%(P<0.05)$ was considered significant.

\section{Results}

\section{Sev caused cognitive deficiency by inhibiting oligodendrocyte growth and myelin sheath formation}

We treated mice 14 days after birth with $3 \%$ sevoflurane for $6 \mathrm{~h}$. Cognitive function of mice was detected by water maze test 14 days after anesthesia. Theresult showed that escape latency increased, but platform crossing time and time spent in target quadrant of Sev group decreased compared with that of control group, indicating that Sev caused the cognitive deficiency in infant mice as shown in figure $2 \mathrm{~A}, \mathrm{~B}$, C, D, and E. Myelin played an important role in learning and memory[6]. Myelin basic protein (MBP), which mainly arose from the gene in the oligodendrocyte, was essential to formation of the multilamellar myelin sheath of the mammalian central nervous system (CNS)[19]. In order to investigate the relationship between the cognitive deficiency and myelin sheath formation, we detectedthe expression of MBP and oligodendrocyte growth. We found that Sev dramatically downregulated the MBP expression and oligodendrocyte growth as shownin Figure 2F, G, H, and I in hippocampus and cortex. We further found that these effects of Sev neurotoxicity on oligodendrocyte were due to the iron deficiency induced by Sev as shown in Figure $2 \mathrm{~J}$ and $\mathrm{K}$. 


\section{Sev induced iron deficiency in neurons}

Hippocampus and cortex were responsible for the cognitive function. In order to evaluate the effect of Sev on the iron homeostasis in hippocampus and cortex, firstly, we tested the expression of FtH, FtL, and TfR1 of hippocampus and cortex. The results showed that Sev significantly decreased the FtH and FtL, but increased the TfR1, meaning that Sev caused the iron deficiency in both tissues as shown in Figure 3 $A, B, C$, and D. Perl's staining also confirmed that Sev indeed lowed the iron levels in hippocampus and cortex as shown in Figure $3 \mathrm{E}, \mathrm{F}, \mathrm{G}$ and $\mathrm{H}$. Iron levels of whole hippocampus and cortex tissues could not reflect the iron levels difference of the different all kinds of nerve cells. To evaluate the iron concentration in neurons, we assayed the FtH using immunofluorescence double-labeling method. The results showed that Sev significantly upregulated the expression of FtH in neurons of hippocampus and cortex, indicating that Sev damaged function of neurons by causing iron decrease as shown in Figure $3 \mathrm{l}$ and $\mathrm{J}$.

\section{Sev affected the NPCs proliferation in hippocampus and cortex}

Iron was a cofactor of ribonucleotide reductase which played the role of DNA synthesis. Considering the role of cell proliferation of iron, we used BrdU staining to investigate the DNA synthesis in hippocampus and cortex induced by Sev. Our results showed that Brdu-labeled positive cells were significantly reduced in hippocampus and cortex, indicating that Sev significantly inhibited the DNA synthesis in brain compared with that of control group as shown in Figure 4 A. Ki67 was a biomarker representing cell proliferation, and Pax6 was a transcription factor that was expressed in NPCs during neurogenesis specifically of the developing central nervous system[20]. In order to further illuminate the influence of Sev on the proliferation of NPCs in hippocampus and cortex, we tested the expression of Ki67 and Pax6. Our results revealed a decrease in the proportion of Ki67+ cells and Pax6+ cells, indicating that iron deficiency induced by Sev may be one of the reasons for neurodevelopment as shown in Figure 4B and C.

\section{Sev caused the iron decrease in NSCs and inhibited NSCs proliferation}

NPCs derived from NSCs. In the ventricular zone/subventricular zone (VZ/SVZ) of cortex and DG zone of hippocampus, NSCs /NPCs continuously proliferate and produce new neurons. To evaluate the effects of Sev on iron levels and proliferation of NSCs, we investigated the expression of FtH, FtL TfR1 and cell cycle in NSCs line NE4C in vitro. We found that Sev similarly up-regulated the expression of TfR1, downregulated the expression of $\mathrm{FtH}$ and $\mathrm{FtL}$, meanwhile, inhibited the cell division when the NE4C cells were treated with Sev for $6 \mathrm{~h}$ as shown in Figure $5 \mathrm{~A}$ and $\mathrm{B}$, indicating that Sev also induced the iron deficiency in NSCs. Decreasd of iron in NE4C further inhibited NSCs proliferation. The number of cells in $4 \mathrm{~N}(16.6 \%)$ was significantly lower than that(37.1\%) of the control group, indicating that Sev inhibited the NSCs proliferation as shown in Figure $5 \mathrm{C}$. It took 28 days for NSCs to differentiate into other nerve cells[21]. In order to verify the cognitive effect of NSCs differentiation induced by Sev, we performed WMW experiments to test the cognitive abilities of the infant mice, when the mice were anesthetized for 28 days. The results showed that the mice were still characterized by cognitive deficits as shown in Figure 5 $D, E, F, G$ and $H$, indicating that Sev-induced cognitive impairment maybe had long-term effect through affecting NSCs proliferation and differentiation. NeuN, CC1, IBA1 and GFAP were the markers of neurons, 
oligodendrocytes, microglia and astrocytes, respectively. To investigate the effects of Sev on these cells after the mice were anesthetized for 28 days, we assayed the expression of these proteins in hippocampus and cortex. Our results showed that only caused decrease of NeuN in hippocampus and did not influence the expression of other proteins in hippocampus and cortex, indicating that Sev maybe affected the function of neurons in hippocampus as shown in Figure $5 \mathrm{I}, \mathrm{J}, \mathrm{K}$ and $\mathrm{L}$.

\section{Iron supplementation inhibited the neural toxicity induced by Sev}

Given the neurotoxic effects of iron deficiency induced by Sev, iron supplementation was performed on the day of pregnancy, and ferrous gluconate solution $(0.1 \mathrm{mg} / \mathrm{ml})$ was added to the drinking water of female mice until the 14th day of offspring birth. We ran further tests and found that iron supplementation dramatically improved the cognitive deficiency in young mice as shown in Figure 6 A, B, $C$, and D. Meanwhile, iron supplementation also significantly alleviated Sev-induced iron deficiency and iron metabolism imbalance in hippocampus and cortex as shown in Figure 6E, F, G, H, I, J, and K, indicating that iron supplementation before Sev anesthesia may be an effective strategy to inhibit Sevinduced neurotoxicity.

\section{Iron therapy restored iron levels in neurons and oligodendrocytes and rescued the proliferation ability of NPCs and the integrity of myelin sheath}

In order to further investigate the mechanism of iron supplementation reducing Sev induced neurotoxicity, we assayed the expression of FtH and FtL in neurons and oligodendrocytes, MBP and Pax6 in cortex and hippocampus, using immunofluorescence or western blot. Our results showed that iron therapy significantly compensated for Sev-induced iron deficiency in mature neurons and oligodendrocytes of cortex and hippocampus as shown in Figure $7 \mathrm{~A}, \mathrm{~B}, \mathrm{C}$, and D. Meanwhile, iron therapy also rescued the decreased expression of MBP in cortex and hippocampus induced by Sev as shown in figure $7 \mathrm{E}, \mathrm{F}, \mathrm{G}$ and $\mathrm{H}$. Moreover, iron therapy also inhibited Sev-induced injury to myelin development and protected the function of neurons.

\section{Discussion}

Anesthesia was irreplaceable in clinical operation. However, anesthesia could induce neural toxicity causing cognitive dysfunction. For example, postoperative cognitive dysfunction (POCD) was a major clinical issue induced by anesthetics especially in the aged patients [14, 22]. In recent years, the toxic side effects of anesthetics on infants' brains had also been widely concerned [2, 8, 23], but the exact mechanism is not clear. Our previous study had shown that Sev could affect postnatal cognitive function through maternal induction of fetal brain iron metabolism disorder in mice [6]. In this study, we investigated the mechanism and effect of Sev on the cognitive function with postnatal 14 days mouse model to mimic the anesthesia operation of infants in the clinic. Our result showed that escape latency, platform crossing time, and time spend in target quadrant all decreased after the infant mice exposed to $2 \%$ Sev for $6 \mathrm{~h}$, indicating the cognitive function of mice appeared deficient (Fig. 2.A, B and C). Oligodendrocytes are the main cells of myelination [24], and myelin development was closely related to 
learning and cognitive function[25]. CC1 was the marker molecular of oligodendrocytes [26]. MBP is one of the most abundant structural proteins of myelin, and its expression levels reflect the status of myelinization. To investigate the effect of Sev on the oligodendrocytes development and myelination, we tested the expression of MBP and CC1 in hippocampus and cortex. We found that Sev dramatically inhibited the myelination and reduced the number of oligodendrocytes, indicating that Sev supressed the development of oligodendrocytes (Fig. 2E-L). Given the important role of iron on the neural development [27], we assayed the FtL expression of oligodendrocytes. Our results showed that Sev decreased the FtL expression indicating that iron deficiency Sev-induced might be one of the important mechanisms of inhibiting myelination and the oligodendrocytes development(Fig. 2M and N). Hippocampus and cortex tissues were the maily brain regions responsible for the cognitive ability. Further, We examined the effect of Sev on iron levels of whole hippocampal and cortex tissues. Our results showed that Sev dramatically down-regulated the FtH, FtL expression and up-regulated the TfR1 expression in hippocampus and/or cortex, indicating that Sev also caused the iron deficiency in hippocampus and cortex as shown in Fig. 3A-D which was consistent with Perl's iron staining results (Fig. 3E and F). Considering the role of neurons in cognitive function, we further investigated the iron levels of neurons in hippocampus and cortex. Our results showed that caused the iron deficiency of neurosn in hippocampus and cortex similarly as shown in Fig. $3 \mathrm{I}$ and $\mathrm{J}$.

Ki67 and Pax6 were one of the markers in cell proliferation and neurogenesis and expressed highly in NPCs. NPCs played the important role in nerve cell proliferation and neurogenesis. In order to evaluated the effect of Sev on the NPCs proliferation, we tested the DNA synthesis, expression of Ki67 and Pax6 with BrdU staining and immunofluorescence assay. We found that iron deficiency induced by Sev suppressed DNA replication afterward decreasing the expression of Ki67 and Pax6 in hippocampus and cortex (Fig. 4A, B and C), indicating that Sev inhibitied the NPCs proliferation. NSCs/NPCs were the progenitor cells for the growth and development of various nerve cells. In order to investigate the effect of Sev on NSCs, we further tested the expression of FtL, FtH, and TfR1 as well as cell cycle of NE4C which was a kind of NSC from hippocampus in vitro. Interestingly, we found that Sev also caused the iron deficiency of NE4C cells, and cells of $4 \mathrm{~N}$ significantly decreased compared with that of control group (Fig. 4D, E, and F). It took 28 days for NSCs to differentiate into other nerve cells. We continued to monitor the cognitive performance of the infant mice 28 days after anesthesia. The results showed that the mice still appeared cognitive deficiency (Fig. 5D-H), indicating that iron metabolism dysfunction of NSCs/NPCs induced by Sev affected the nerve development. It probably maily affected the development and function of neurons in hippocampus (Fig. 5I-L) rather than cortex. Since Sev could cause iron deficiency, we administered iron supplementation before anesthesia of mice. Interestingly, our results showed that these damage effects induced by Sev could be significantly eliminated by iron supplementation pretreatment including iron deficiency in hippocampus, cortex, neurons, and oligodendrocyte. Iron therapy also rescued the cognitive impairment, hypomyelination and inhibition of cell proliferation of NSCs/NPCs induced by Sev. as shown in Fig. 6 and Fig. 7.

\section{Conclusion}


These data suggest that Sev-induced iron deficiency may be one of the important mechanisms of cognitive impairment caused by inhaled anesthetics in mice. Iron supplementation before anesthesia is an effective strategy to prevent cognitive impairment caused by Sev in infants.

\section{Abbreviations}

Sev, sevoflurane; TfR1, transferrin receptor 1; FtH, ferritin heavychain; FtL, ferritin lightchain; MBP, myelin basic protein; NSCs, neural stem cells ; NPCs, neural progenitor cells; BrdU, bromodeoxyuridine; MWM, Morris water maze

\section{Declarations}

\section{Acknowledgments}

This work was supported by Hebei Province Science Foundation (grant numbers C2020205036 and C2019205262), and Hebei Normal university foundation (grant numbers L2019Z07 and L2019B27),

\section{Data availability}

The datasets are available from the corresponding author on reasonable request.

\section{Authors' contributions}

Jianhua Zhang and Zhenhua Shi conceived and designed the experiments. Jinhong Xie, Yong Zuo, Xue Zhang and Di Zhang Anand Thirupathi, performed the experiments. Yong Zuo and Jinhong Xie completed the statistical analysis of data. Anand Thirupathi and Zhen-Hua Shi wrote the manuscript.

\section{Conflicts of Interest}

The authors declare that they have no competing interests.

\section{References}

1. McCann ME, de Graaff $J$ (2017) Current thinking regarding potential neurotoxicity of general anesthesia in infants. Curr Opin Urol 27:27-33

2. Yu Y, Yang Y, Tan H, Boukhali M, Khatri A, Yu Y, Hua F, Liu L, Li M, Yang G, Dong Y, Zhang Y, Haas W, Xie Z (2020) Tau Contributes to Sevoflurane-induced Neurocognitive Impairment in Neonatal Mice, Anesthesiology, 133 595-610

3. Sun L (2010) Early childhood general anaesthesia exposure and neurocognitive development. $\mathrm{Br} \mathrm{J}$ Anaesth 105(1):i61-68

4. Feng YP, Yang TS, Chung CH, Chien WC, Wong CS (2020) Early childhood general anesthesia exposure associated with later developmental delay: A national population-based cohort study. PLoS ONE 15:e0238289 
5. Backeljauw B, Holland SK, Altaye M, Loepke AW (2015) Cognition and Brain Structure Following Early Childhood Surgery With Anesthesia, Pediatrics, 136 e1-12

6. Zuo Y, Li B, Xie J, Ma Z, Thirupathi A, Yu P, Gao G, Zhou J, Zhou C, Xu H, Chang Y, Shi Z (2020) Sevoflurane anesthesia during pregnancy in mice induces cognitive impairment in the offspring by causing iron deficiency and inhibiting myelinogenesis. Neurochem Int 135:104693

7. Wu Z, Xue H, Gao Q, Zhao P (2020) Effects of early postnatal sevoflurane exposure on oligodendrocyte maturation and myelination in cerebral white matter of the rat. Biomed Pharmacother 131:110733

8. Dai CL, Li H, Hu X, Zhang J, Liu F, Iqbal K, Gong CX (2020) Neonatal Exposure to Anesthesia Leads to Cognitive Deficits in Old Age: Prevention with Intranasal Administration of Insulin in Mice. Neurotox Res 38:299-311

9. Andropoulos DB (2018) Effect of Anesthesia on the Developing Brain: Infant and Fetus. Fetal Diagn Ther 43:1-11

10. Georgieff MK (2020) Iron deficiency in pregnancy. Am J Obstet Gynecol 223:516-524

11. Galaris D, Barbouti A, Pantopoulos K (2019) Iron homeostasis and oxidative stress: An intimate relationship. Biochim Biophys Acta Mol Cell Res 1866:118535

12. Xu G, Lu H, Dong Y, Shapoval D, Soriano SG, Liu X, Zhang Y, Xie Z (2017) Coenzyme Q10 reduces sevoflurane-induced cognitive deficiency in young mice. Br J Anaesth 119:481-491

13. Wang M, Zuo Y, Li X, Li Y, Thirupathi A, Yu P, Gao G, Zhou C, Chang Y, Shi Z (2021) Effect of sevoflurane on iron homeostasis and toxicity in the brain of mice. Brain Res 1757:147328

14. Ge X, Zuo Y, Xie J, Li X, Li Y, Thirupathi A, Yu P, Gao G, Zhou C, Chang Y, Shi Z (2021) A new mechanism of POCD caused by sevoflurane in mice: cognitive impairment induced by crossdysfunction of iron and glucose metabolism. Aging 13:22375-22389

15. Gao Y, Ma L, Han T, Wang M, Zhang D, Wang Y (2020) Protective role of protocatechuic acid in sevoflurane-induced neuron apoptosis, inflammation and oxidative stress in mice. Restor Neurol Neurosci 38:323-331

16. Xu L, Shen J, Yu L, Sun J, McQuillan PM, Hu Z, Yan M (2018) Role of autophagy in sevofluraneinduced neurotoxicity in neonatal rat hippocampal cells. Brain Res Bull 140:291-298

17. Liu S, Fang F, Song R, Gao X, Jiang M, Cang J (2018) Sevoflurane affects neurogenesis through cell cycle arrest via inhibiting wnt/beta-catenin signaling pathway in mouse neural stem cells. Life Sci 209:34-42

18. Wang K, Tian Y, Zhang Y, Li X, Wei X, Hu H, Xu S (2019) Toxicity mechanism of sevoflurane in neural stem cells of rats through DNA methylation. Exp Ther Med 18:237-241

19. Vassall KA, Bamm VV, Harauz G (2015) MyelStones: the executive roles of myelin basic protein in myelin assembly and destabilization in multiple sclerosis. Biochem J 472:17-32

20. Hasan SM, Sheen AD, Power AM, Langevin LM, Xiong J, Furlong M, Day K, Schuurmans C, Opferman JT, Vanderluit JL (2013) Mcl1 regulates the terminal mitosis of neural precursor cells in the 
mammalian brain through p27Kip1, Development, 140 3118-3127

21. Braun SM, Jessberger $S$ (2014) Adult neurogenesis: mechanisms and functional significance. Development 141:1983-1986

22. Safavynia SA, Goldstein PA (2018) The Role of Neuroinflammation in Postoperative Cognitive Dysfunction: Moving From Hypothesis to Treatment. Front Psychiatry 9:752

23. Rosenblatt A, Kremer M, Swanson B, Shah R (2019) Anesthesia Exposure in the Young Child and Long-term Cognition: An Integrated Review. AANA J 87:231-242

24. Kuhn S, Gritti L, Crooks D, Dombrowski Y (2019) Oligodendrocytes in Development, Myelin Generation and Beyond. Cells, p 8

25. Park JH, Choi HY, Cho JH, Kim IH, Lee TK, Lee JC, Won MH, Chen BH, Shin BN, Ahn JH, Tae HJ, Choi JH, Chung JY, Lee CH, Cho JH, Kang IJ, Kim JD (2016) Effects of Chronic Scopolamine Treatment on Cognitive Impairments and Myelin Basic Protein Expression in the Mouse Hippocampus. J Mol Neurosci 59:579-589

26. Wang J, He X, Meng H, Li Y, Dmitriev P, Tian F, Page JC, Lu QR, He Z (2020) Robust Myelination of Regenerated Axons Induced by Combined Manipulations of GPR17 and Microglia. Neuron 108:876$886 \mathrm{e} 874$

27. Bastian TW, von Hohenberg WC, Georgieff MK, Lanier LM (2019) Chronic Energy Depletion due to Iron Deficiency Impairs Dendritic Mitochondrial Motility during Hippocampal Neuron Development. J Neurosci 39:802-813

\section{Figures}




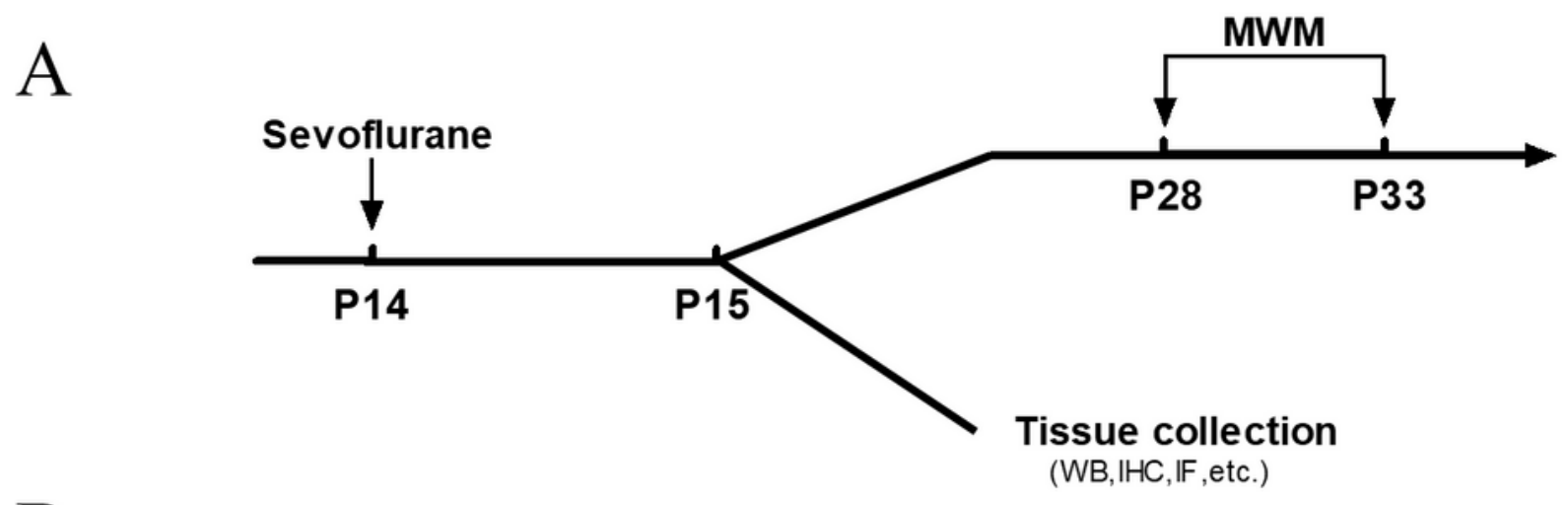

B

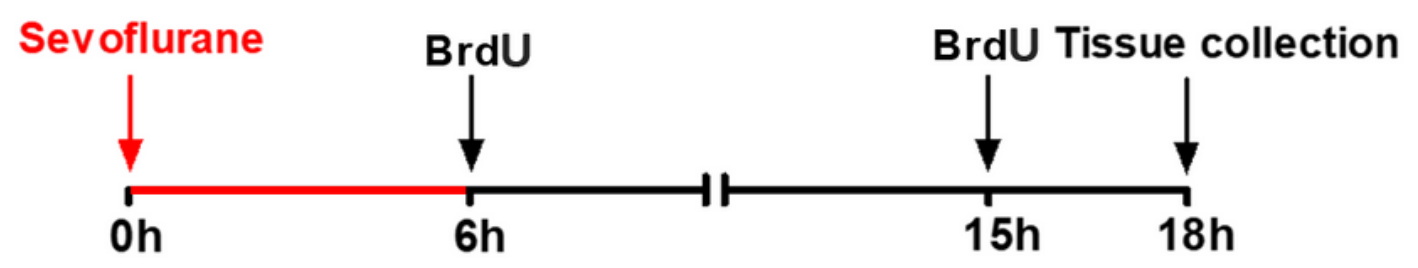

$\mathrm{C}$

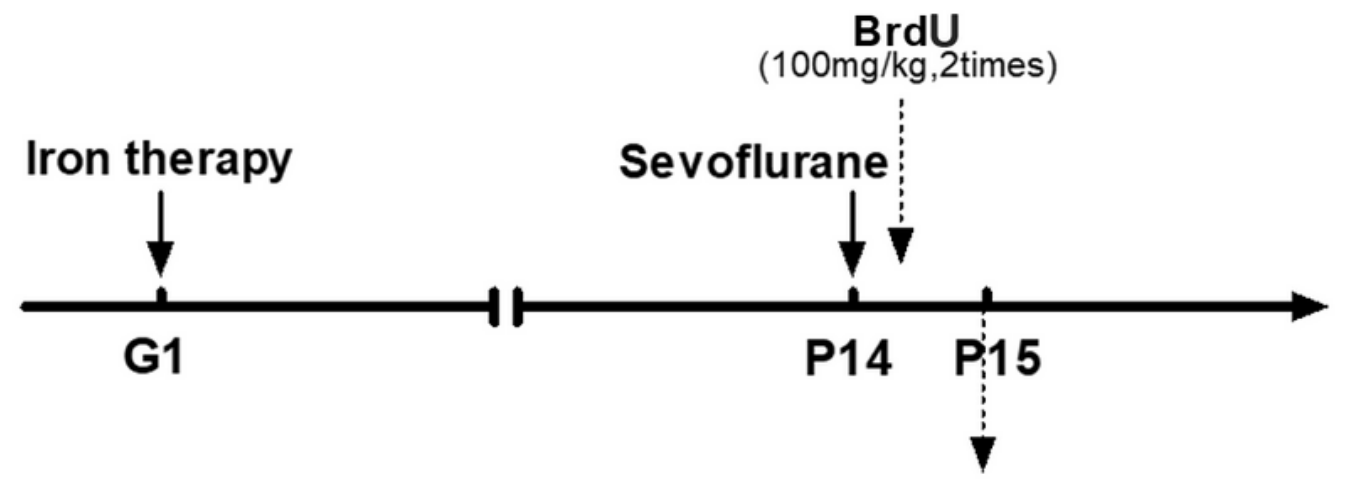

Tissue collection

(WB, $\mathrm{HC}, \mathrm{IF}$, etc. $)$

$\mathrm{D}$

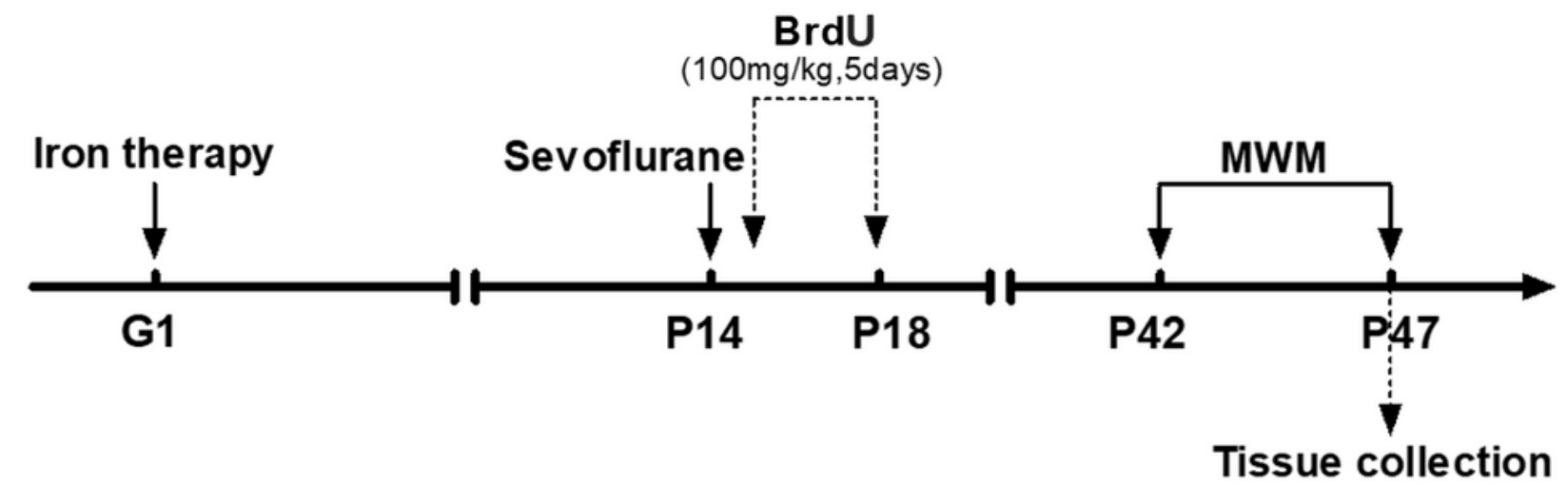

Figure 1

Schematic diagram of experimental design.

A. The mice of postnatal day $14(\mathrm{P} 14)$ were treated with Sev for $6 \mathrm{~h} .12$ hours after the anesthesia, the mice were divided into two groups randomly. One group of mice was used to evaluate the cognitive function by MWM when the mice of postnatal day 28. Another group of mice was harvested to assay the 
protein expression levels. B. The mice of postnatal day 14(P14) were treated with Sev for 6h. After anesthesia, the mice were immediately treated with $\mathrm{BrdU}(100 \mathrm{mg} / \mathrm{Kg}$, intraperitoneal injection). After $9 \mathrm{~h}$, the mice were treated with BrdU again. After $3 \mathrm{~h}$, the brain tissues were harvested to run a follow-up experiment. C. On their first day of pregnancy (G1), the mice were fed with drinking

water containing gluconate solution $(0.1 \mathrm{mg} / \mathrm{ml})$. The next step was the same

as A and B. D. It took 28 days for NSCs to differentiate into other nerve cells. To evaluate the effect of Sev on the differentiation of NSCs, the iron and BrdU treatment were as same as B and C. 28 days after Sev treatment, MWM tested the cognitive function of mice.

A

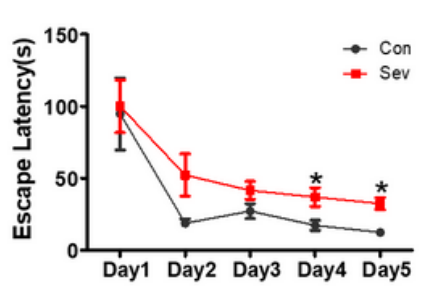

$\mathrm{C}$
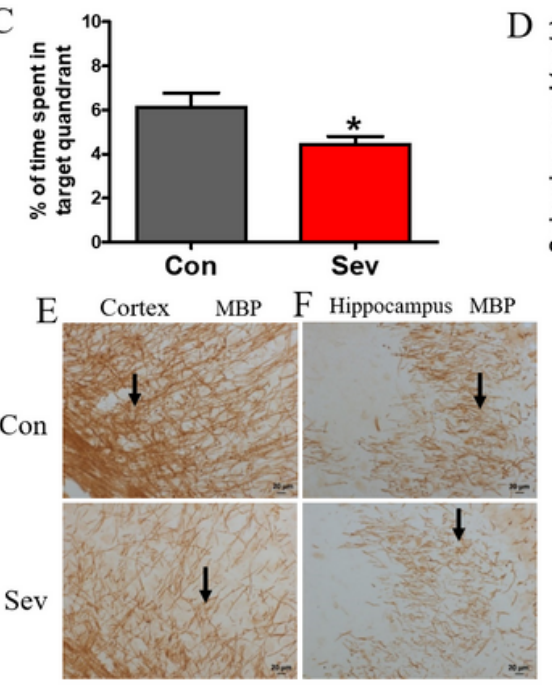

$\mathrm{B}$

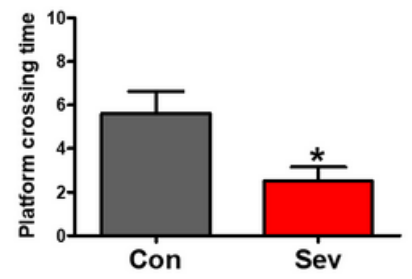

D
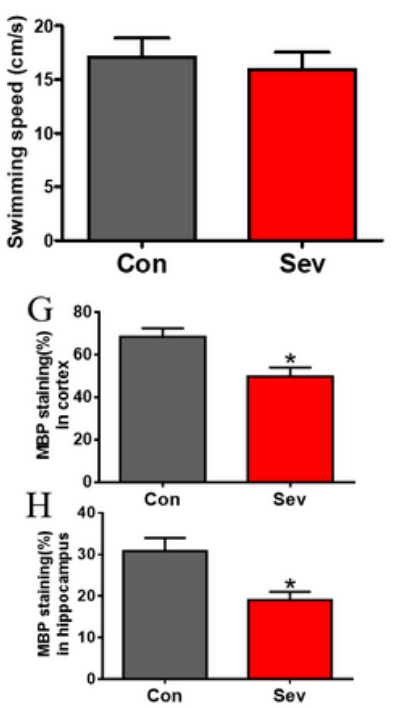
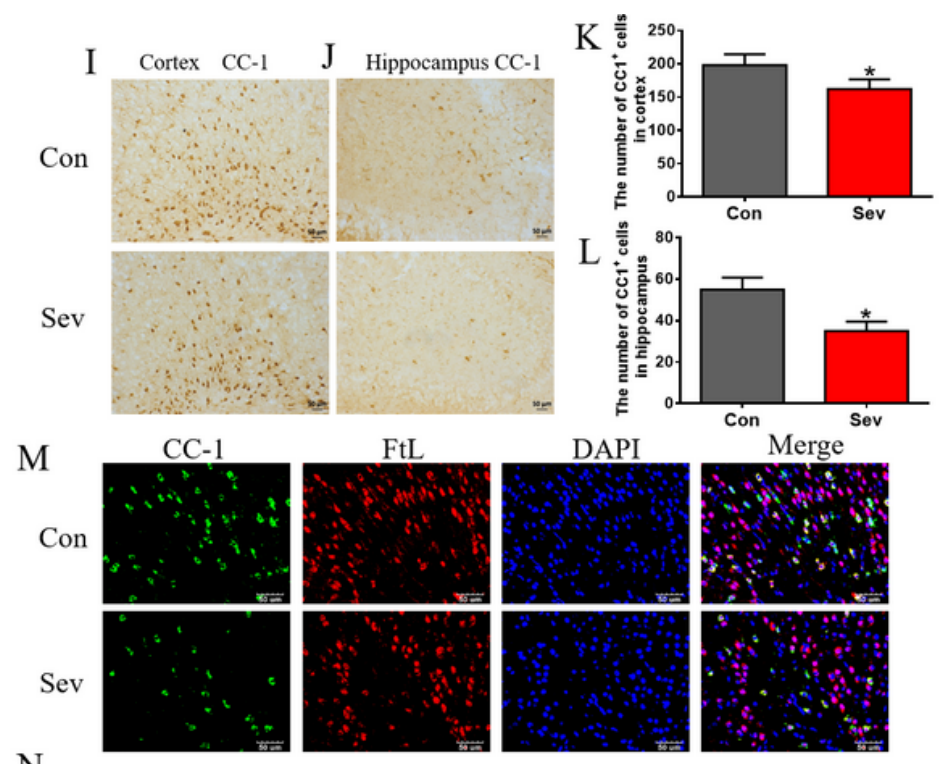

$\mathrm{N}$
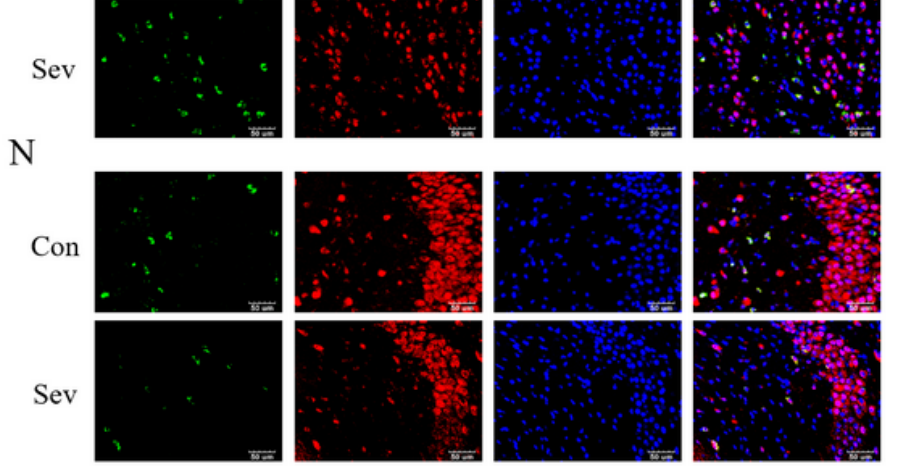

\section{Figure 2}

The effects of Sev on cognitive function, MBP and CC1 expression in hippocampus and cortex, and FtL expression in oligodendrocytes.

The mice were treated with Sev as described in the materials and methods section. The cognitive function was detected by the MWM test $(A, B, C$ and $D)$. The results were calculated as the escape latency (sec.) and platform crossing time \pm SEM $(n=15)$. E-L showed the expression of MBP and CC1 in hippocampus and cortex using immunohistochemistry $(n=6), * P<0.05$ compared with the control group by t-test. 

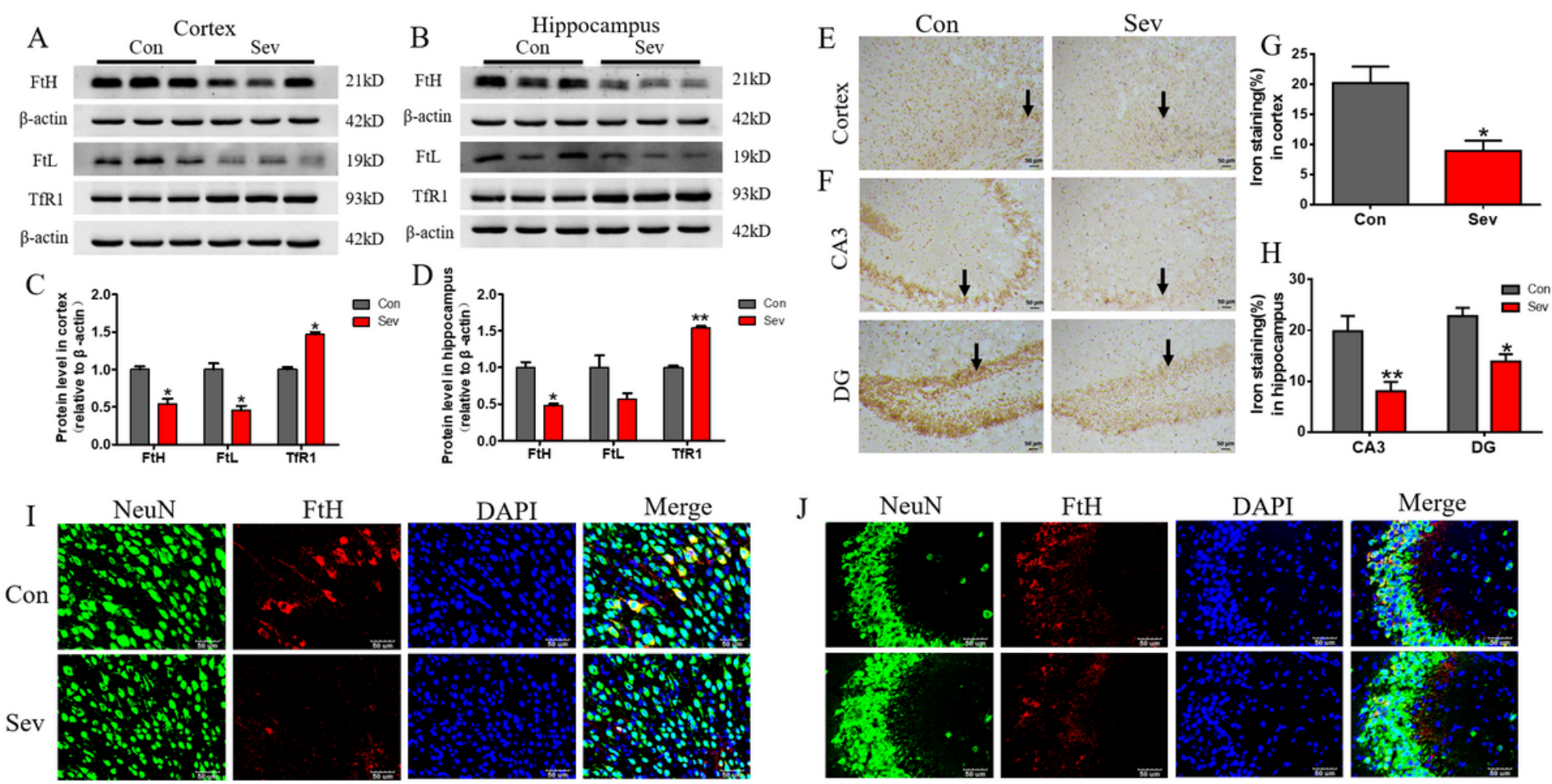

DAPI
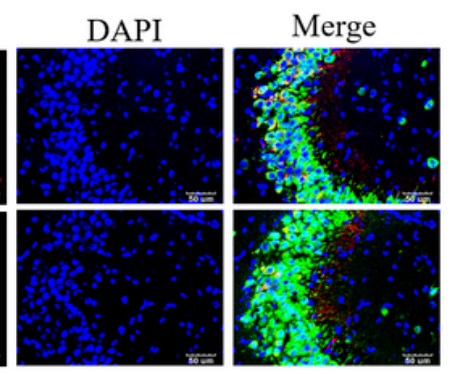

Figure 3

The effects of Sev on expression of FtH, FtL and TfR1 in hippocampus and cortex or neurons, as well as iron staining in hippocampus and cortex.

A-D showed the expression of FtH, FtL, and TfR1 in hippocampus and cortex. E-H showed the iron levels in hippocampus and cortex using the Perl's staining. I and $\mathrm{J}$ showed the expression of FtH in neurons from hippocampus and cortex. Data are expressed as mean \pm SEM $(n=6)$, ${ }^{*}<0.05$ and ${ }^{\star \star} P<0.01$, respectively, compared with the control group by t-test..
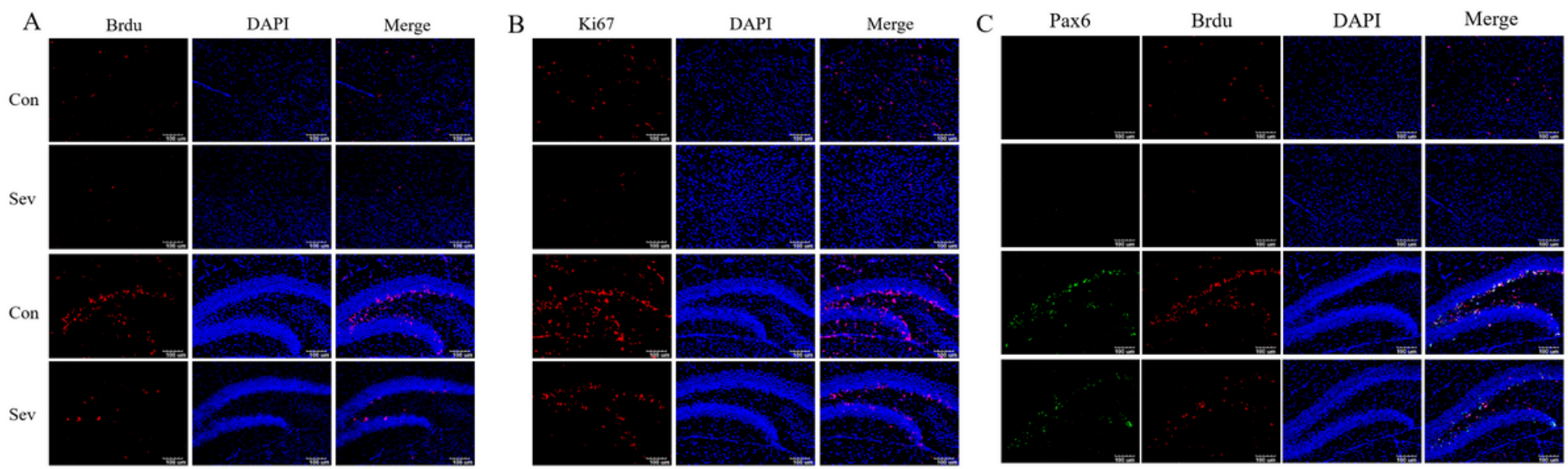

Figure 4

Sev inhibited the DNA synthesis and the NPCs proliferation in cortex and hippocampus 
A showed that Sev inhibited the DNA synthesis using BrdU staining in cortex and hippocampus. B and C were expressions of Ki67 and Pax6 in NPCs

using immunofluorescence assay respectively
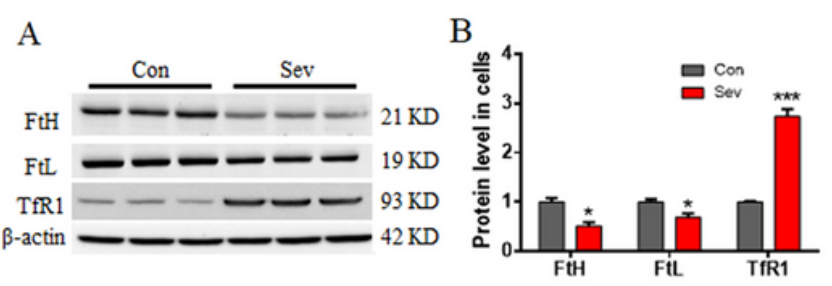

$\mathrm{D}$

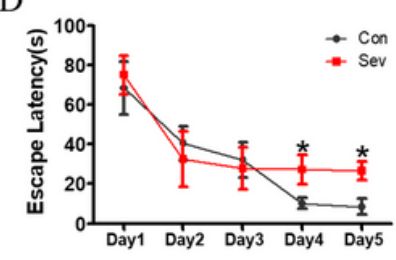

$\mathrm{E}$

F
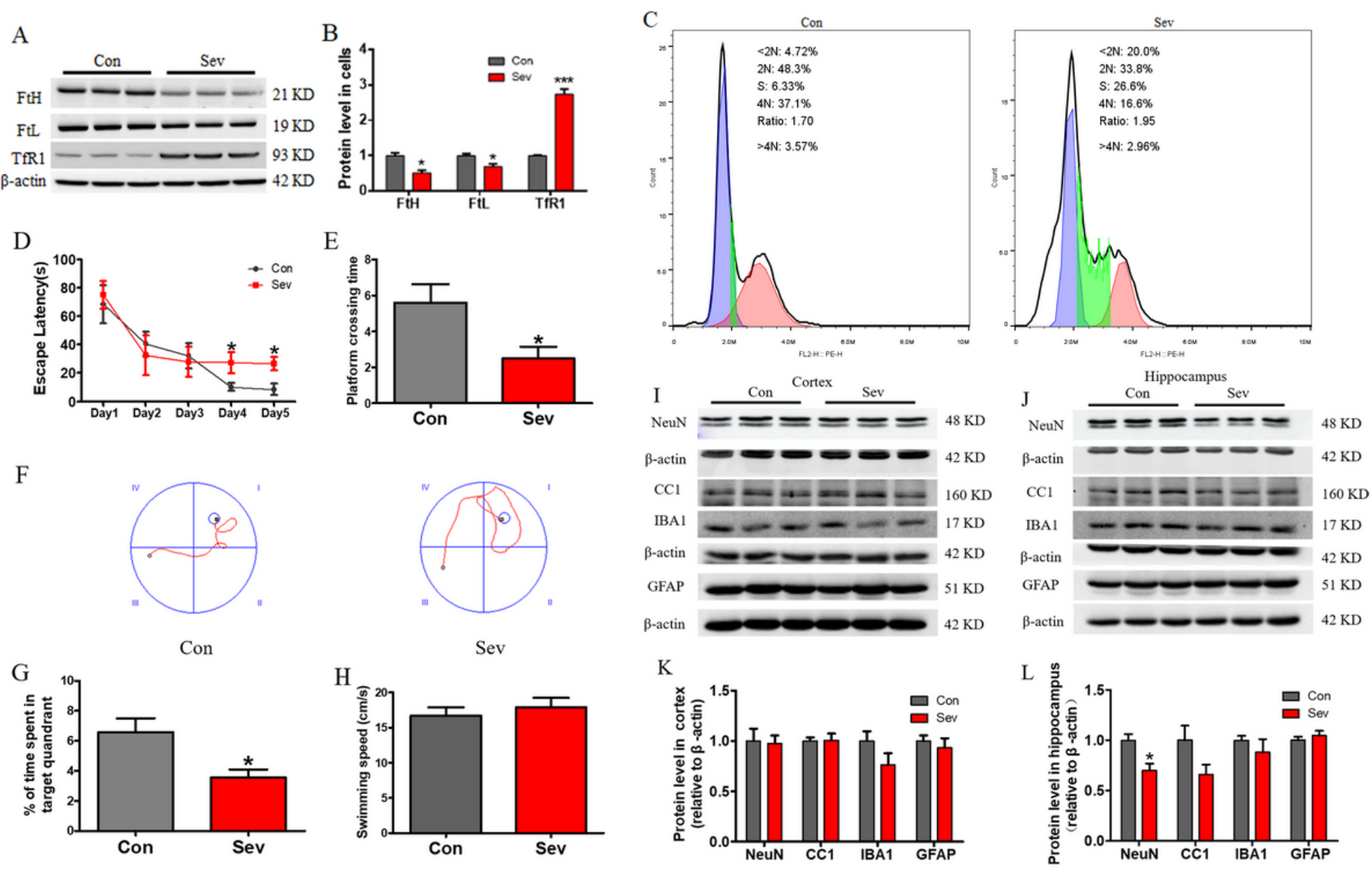

Figure 5

The effects of Sev on iron leves, cell proliferation of NSC and, cognitive function after $\mathbf{2 8}$ days of Sev treatment.

A and B showed the expression of FtH, FtL and TfR1 in NE4C cells. C represented the effect of Sev on cell proliferation of NE4C cells by flow cytometry. F-H showed the cognitive function test after 28 days of Sev treatment. I-L showed the effects of Sev on neurons, oligodendrocyte and microglial cells by detecting biomarker molecule expression using western blot in hippocampus and cortex. Data are expressed as mean $\pm \operatorname{SEM}(n=6),{ }^{*}<0.05$ compared with the control group by t-test.. 


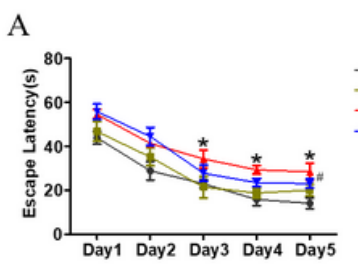

B
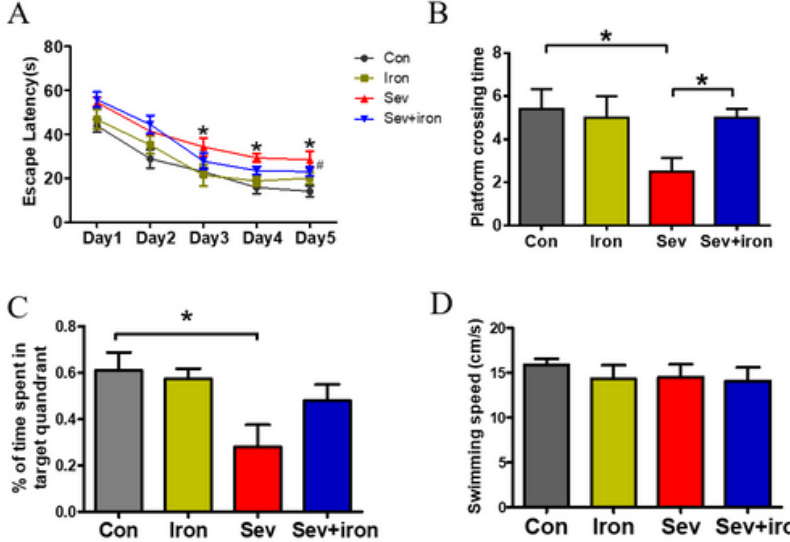

D

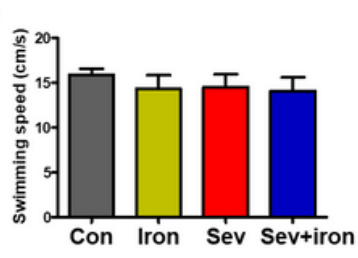

Cortex
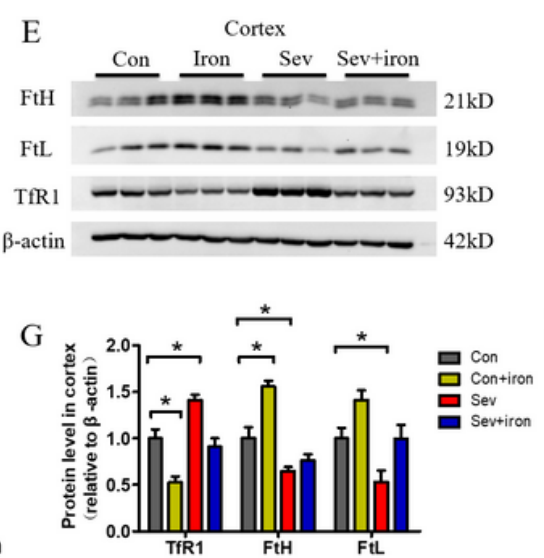

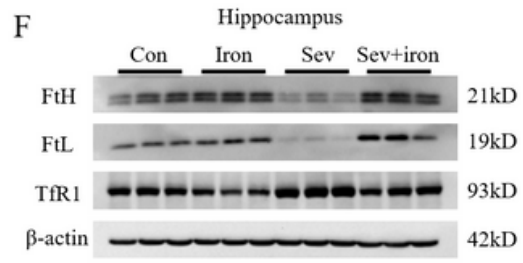

$\mathrm{H}$

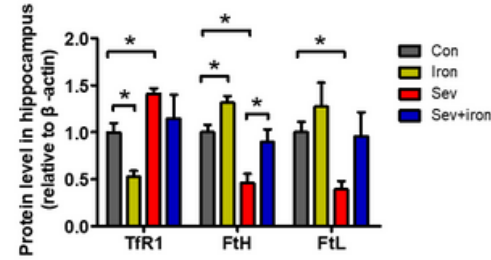

$\mathrm{K}$

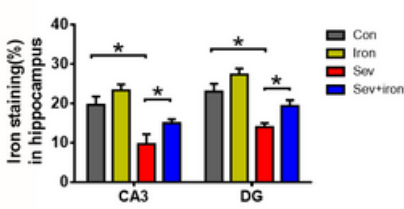

Figure 6

Iron supplementation inhibited the iron deficiency in brain and rescued the cognitive dysfunction induced by Sev

A-D showed the cognitive function testing by morris water maze. E-H showed the expression of FtH, FtL, and TfR1 in hippocampus and cortex induced by Sev after iron supplementation. I-K showed the iron levels in hippocampus and cortex induced by Sev after iron supplementation. Data are expressed as mean \pm SEM $(n=6),{ }^{*} P<0.05$ and ${ }^{* \star *} P<0.001$, respectively, compared with the control group by one-way ANOVA.
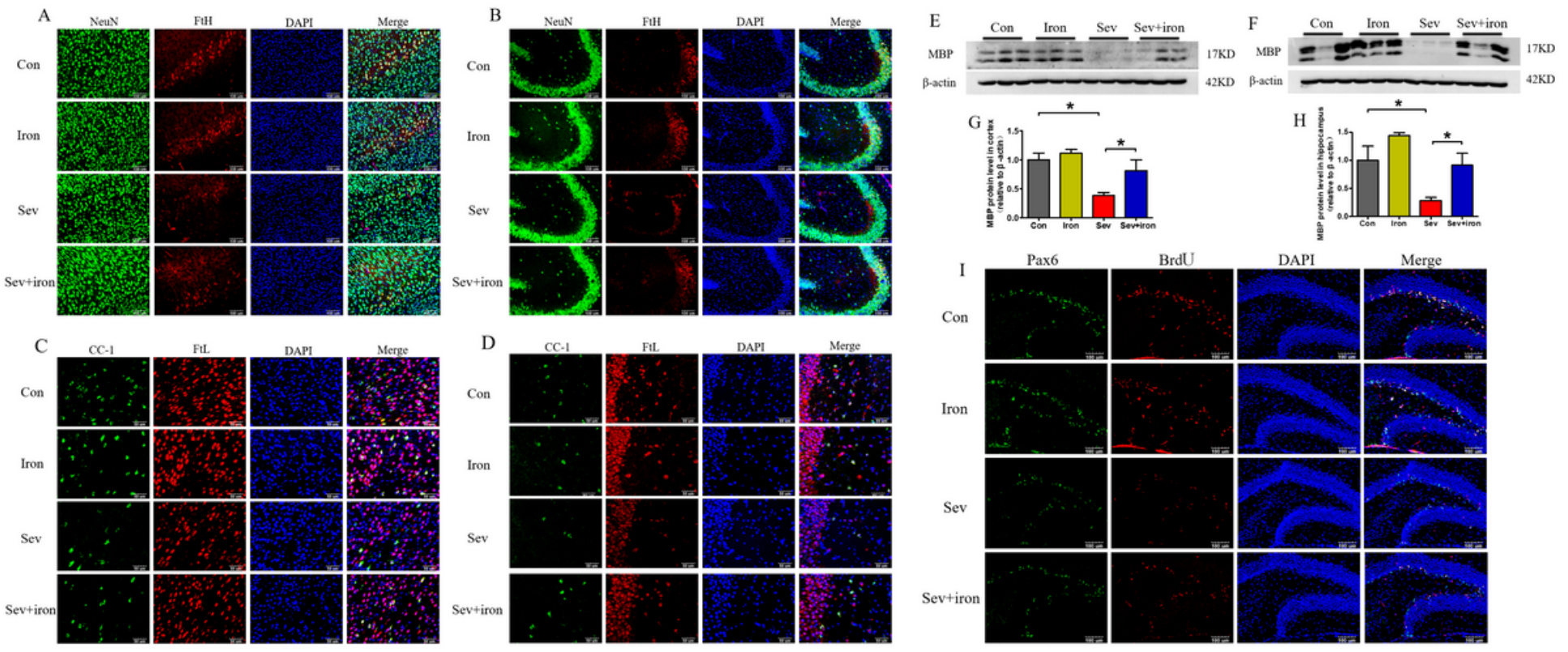

Figure 7 
Iron therapy rescued iron deficiency in nerve cells and oligodendrocytes, and myelin formation.

A -D showed the FtH or FtL expression from hippocampus and cortex in neurons or in oligodendrocytes by using immunofluorescence. E-H showed the MBP expression induced by Sev after iron therapy. I showed the Pax6 expression in hippocampus and cortex induced by Sev after iron therapy. Data are expressed as mean $\pm \operatorname{SEM}(n=6),{ }^{*} P<0.05$ compared with the control group by one-way ANOVA. 